\title{
Effet des teneurs en grain et de la variabilité génétique sur la valeur énergétique du maïs ensilage mesurée par des vaches laitières
}

\author{
Y Barrière *, JC Émile \\ avec la collaboration de $R$ Traîneau, L Huguet, $M$ Lila \\ INRA, station d'amélioration des plantes fourragères, 86600 Lusignan, France
}

(Reçu le 24 mai 1989 ; accepté le 10 janvier 1990)

\begin{abstract}
Résumé - Alors que se mettent en place d'importants programmes de sélection du maïs destiné à l'ensilage de la plante entière, incluant la prise en compte de critères de digestibilité in vitro, des expérimentations ont été réalisées sur vaches laitières afin de déterminer l'importance des teneurs en grain et de la variabilité génétique du maïs sur la production du lait. La comparaison d'hybrides normalement ensilés, ou appauvris du 1/8 ou du 1/4 de leurs épis le jour de la récolte, montre qu'il n'y a pas d'effet des teneurs en grain entre 41 et $48 \%$ sur la production de lait à $4 \%$. Pour chacun de ces essais les variations de poids des animaux de chacun des lots sont très semblables. Les 3 comparaisons successives de 2 génotypes de précocité similaire ne mettent en évidence qu'une seule fois un effet significatif de la variété de maïs sur la production de lait brut, avec un écart de production journalière entre ces 2 variétés de 1,5 kg. Avec la gamme de variabilité étudiée ici, aucune des méthodes habituelles de test de la digestibilité ou de la valeur énergétique ne semble permettre une prédiction complètement satisfaisante des résultats observés avec les vaches laitières. Les digestibilités de la matière organique et les valeurs énergétiques de référence (UFL), obtenues sur moutons standard, sous-estiment la valeur des ensilages pauvres en grain. Les digestibilités estimées par la méthode de Tilley et Terry, comme la solubilité enzymatique employée, ne permettent pas de retrouver clairement les caractéristiques des ensilages mises en évidence par les vaches laitières, avec en particulier, un tassement des écarts observés. L'idéotype maïs fourrage pourrait donc être une plante permettant de récolter, à une teneur en matière sèche proche de $30 \%$, une forte quantité de biomasse, dont la teneur en grain pourrait être de 5 à 10 points inférieure à celle des hybrides actuels, avec une bonne digestibilité des plantes entières dans la ration, associée à une très bonne résistance à la verse en végétation.
\end{abstract}

maïs ensilage / digestibilité / vache laitière / variabilité génétique / valeur énergétique

Summary - Silage corn : effect of grain content and genetic variability on milk yield of dairy cows. There are now important selection programmes in Europe for breeding maize genotypes specific for silage use, with in vitro measurements of feeding value for livestock. To assist breeders, experiments have been performed with dairy cows to determine the effect of grain content and genetic variability on milk production, and the possibility of predicting feed value for dairy cows with standard sheep or in vitro tests. Comparison of normally ensiled hybrids, or ensiled after discarding $1 / 8$ or $1 / 4$ or the ears the day of harvest are discussed in tables III, IV and V. They clearly establish that there is no effect of grain content between 41 and $48 \%$ on milk or fat corrected milk yield, or on silage maize intake. There is also no significant variation in animal body weight Three comparisons of 2 different maize varieties show only once a significant effect of maize genotype on milk yield, with an average daily difference of $1.5 \mathrm{~kg}$ between Dea and Wisconsin 416. With our range of variability, none of the classic tests for predicting digestibility or energetic feeding value of the silage seem very convenient, when compared to values obtained with dairy cows (table VI). Digestibility of organic matter and French fodder unit UFL given by standard sheep, under estimate the value of silage with slight grain content. The Tilley and Terry method and the enzymatic solubility used are not able to clearly explain the variation observed in dairy cows, and lead to a small decrease in the variability between hybrids. Silage maize ideotype could be a hybrid with an important biomass yield having a dry-matter content at harvest close to $30 \%$, and a grain content ranging from 5-10 points lower than actual classic varieties, also having a good digestibility of the whole plant linked to a very good resistance to stalk lodging. However, in relation to the variability in cattle used as test tools, only significant improvement in silage maize digestibility should be taken into consideration.

\section{silage maize / digestibility / dairy cow / genetic variability / feeding value}

\footnotetext{
* Correspondance et tirés à part
} 


\section{INTRODUCTION}

Il y a actuellement dans la CEE près de 3,3 millions d'hectares de maïs ensilage, qui permettent de mettre à la disposition d'animaux à haut potentiel un aliment facile à produire et à conserver, dont la valeur énergétique est élevée et relativement constante. En France, le maïs ensilage représente au moins le quart des surfaces de fourrages semées et cet ensilage constitue la ration de base des animaux pendant une période de plus en plus importante, jusqu'à 10 ou 11 mois par an.

D'une manière générale l'efficacité alimentaire de l'ensilage de maïs est nettement liée à sa teneur en matière sèche, et cela jusqu'à $35 \%$, essentiellement par le biais des quantités ingérées. Les tables de valeur alimentaire (Jarrige, 1988) du maiis ensilage ne prennent d'ailleurs en compte que des effets stades ou conditions de végétation pour spécifier les valeurs nutritives. Telles qu'elles ont pu être établies avec les méthodes de référence in vitro ou par des moutons standard, la digestibilité et surtout la valeur énergétique des ensilages de maïs n'évoluent pratiquement pas entre les stades laiteux et vitreux du grain (Demarquilly, 1969 ; Andrieu et Demarquilly, 1974; Daynard et Hunter, 1975; Gallais et al, 1976; Andrieu, 1984 ; Jarrige, 1988). En se référant aux tables, la valeur énergétique des ensilages de maïs varie de 0,82 à $0,96 \mathrm{UFL} / \mathrm{kg}$ de matière sèche selon les conditions de végétation, et reste stable à 0,90 UFL en conditions normales pour des maïs ayant une teneur en matière sèche comprise entre 25 et $35 \%$.

Une compilation préliminaire de mesures comparatives systématiques de la valeur énergétique de différents hybrides de maiss mesurée par des moutons standard met en évidence un effet génotype significatif, avec, à teneur en matière sèche similaire, un effet de précocité également significatif (Barrière et al, 1988). Mais l'effet du génotype de maïs sur les performances zootechniques d'animaux à haut potentiel est très peu connu, en dehors des études réalisées en comparaison d'hybrides normaux et de leurs formes reconverties brown-midrib-3 (Barrière et al, 1985 ; Hoden et al, 1985 ; Malterre et al, 1985).

De même, le rôle de la teneur en grain dans la valeur énergétique de l'ensilage, pour une teneur en matière sèche donnée, n'est pas clairement établi. D'une part, comme cela a été précisé cidessus, alors que les compositions morphologiques des plantes et leurs teneurs en grain changent notablement entre les stades pâteux et vitreux du grain, la digestibilité et la valeur éner- gétique des ensilages ne semblent que très peu variables pour un génotype donné ; d'autre part, la digestibilité des tiges participe dans la même proportion que la teneur en grain à la digestibilité des plantes entières (Gallais et al, 1981 ; Deinum et Bakker, 1981). Toutefois, la teneur en grain reste dans les mentalités un facteur de qualité de l'ensilage, avec en fait, un risque de confusion entre les effets de teneur en matière sèche et de teneur en grain, qui évoluent dans le même sens. Une équation de prédiction de la valeur énergétique des ensilages de maīs à partir de leurs teneurs en grain a par ailleurs été établie par régression dans un intervalle très large de teneur en grain (25 à 55\%) (Andrieu, 1984), équation dont la précision reste assez limitée $( \pm 0,03$ UFL, $r=0,7)$ même si elle permet une première estimation de la valeur d'un ensilage de maïs, en l'absence d'autres mesures. Enfin les essais de comparaisons d'hybrides de maïs anciens et modernes ont montré que le progrès génétique pour la productivité en grain observé depuis 30 ou 40 ans était dû à une augmentation des transferts de la tige vers l'épi, plutôt qu'à une augmentation de la productivité en biomasse, à indice de récolte constant (Derieux et al, 1987 ; Barrière et al, 1987), avec en outre une amélioration de la tolérance des plantes aux basses températures, aux peuplements plus élevés et une amélioration de la résistance aux verses. Dans une gamme de précocité donnée et pour des hybrides sélectionnés pour leur productivité en grain, il peut donc y avoir une tendance à l'augmentation de la teneur en grain dans des ensilages d'hybrides modernes comparés à des hybrides plus anciens comme Wisconsin 416, INRA 258 ou LG 11.

Les comparaisons, avec des vaches laitières, de l'efficacité alimentaire d'ensilage de maïs en fonction de leur teneur en grain, pour des teneurs en matière sèche données, sont rares. Hemken et al (1971) mettent en évidence des productions de lait semblables, voisines de $17 \mathrm{~kg}$, avec un hybride riche et un hybride pauvre en grain, avec des teneurs en matière sèche de 35,1 et $32,6 \%$ respectivement. Au contraire, Rémond et Journet (1977) montrent que des maïs pauvres en grain (20 à $30 \%$ ) après une saison très sèche conduisaient à une production laitière de 1,7 à $2,4 \mathrm{~kg}$ inférieure à celle permise par des ensilages normaux comparables ( $45 \%$ de grains), les matières sèches étant semblables et comprises entre 30 et $35 \%$. Toutefois on peut noter que, dans ce cas, la variation pour la teneur en grain était très importante (pratiquement du simple au double), et que, d'autre part, les parties non grain des ensilages étaient probablement différentes en ce qui concerne les quan- 
tités et qualités des cellulose, hémicellulose et lignine, conduisant donc à des digestibilités de parois différentes. Enfin, les comparaisons de 2 variétés différentes, ou de densités de semis différentes, faites par l'ITEB (1988-89) montrent que les ensilages les plus pauvres en grain sont légèrement moins bien valorisés, mais, là encore, les parties non grain des différents ensilages n'étaient pas directement comparables.

Les variétés utilisées en ensilage sont encore pour une part importante des variétés issues de la sélection grain. Les travaux réalisés depuis quelques années en France ont permis de créer des variétés plus spécifiquement triées dans des essais de valeur agronomique en plante entière, qui utilisent la base génétique issue de la sélection grain. La productivité en grain de ces variétés est au moins égale à celle des variétés témoins du groupe de précocité considéré (règle d'inscription). A côté d'hybrides mixtes dont la productivité en plante entière est du niveau de celle des témoins, des combinaisons plus originales ont été réalisées avec certaines lignées bien adaptées à la création d'hybrides plus spécifiques pour une utilisation sous forme d'ensilage. Ces lignées se caractérisent par une très bonne aptitude à la combinaison pour la production de biomasse permise par une floraison un peu plus tardive que celle du matériel classique, mais avec une maturation des tiges plus rapide. Le développement d'hybrides de ce type peut être limité en France par leur éventuel plus faible rendement en grain. Mais ils sont plus facilement inscriptibles en Europe du Nord, où le rendement en grain n'est pas pris en compte, mais où, en revanche, la digestibilité des plantes entières est un des critères d'inscription.

Alors que se mettent en place d'importants programmes de création de matériel génétique et de sélection de variétés de maîs spécifiquement destinées à l'ensilage de la plante entière, programmes qui tendent en outre à prendre en compte les caractéristiques de valeur alimentaire, il importe d'une part d'estimer l'expression de la variabilité génétique des ensilages de maïs en terme de performances zootechniques chez des animaux à haut potentiel, et de vérifier la pertinence des critères habituels de mesure de la digestibilité (moutons standard, méthodes Tilley et Terry (1963), ou solubilités enzymatiques (ia, Lila et al, 1986). II importe d'autre part de définir des critères clairs et précis de teneur en grain optimale des ensilages de maïs permettant d'assurer une ration de base de haute valeur énergétique à ces mêmes animaux, sans perdre sur les potentiels génétiques d'amélioration de la productivité du maïs, à précocité constante, mais avec sans doute une teneur en grain plus faible que celle de la plupart des hybrides actuellement cultivés. Cela est d'autant plus nécessaire que les quotas de production imposent de réduire au maximum les coûts intermédiaires de ces productions animales, et que l'amélioration de la productivité et de la qualité des fourrages de base permet d'augmenter les marges des ateliers animaux sans augmenter les productions animales.

Les expérimentations réalisées sur vaches laitières durant les hivers $1986-87,87-88$ et $88-$ 89 à l'INRA de Lusignan permettent d'esquisser des réponses à ces questions, à savoir la valorisation d'une variabilité génétique des ensilages de maïs par des animaux forts producteurs, et l'importance de la teneur en grain de l'ensilage sur sa valeur énergétique, puis d'en tirer les premières conséquences sur les critères de sélection du maïs fourrage.

\section{MATÉRIELS ET MÉTHODES}

Deux variétés de maïs ont été cultivées en 1986, 87 et 88 à l'INRA de Lusignan (Vienne). Elles ont été d'une part ensilées normalement à quelques jours d'intervalle afin d'obtenir des teneurs en matière sèche similaires malgré les faibles différences de précocité des hybrides. Un troisième silo a d'autre part été confectionné avec l'une des 2 variétés, $48 \mathrm{~h}$ après le premier, en éliminant au moment de la récolte 1 épi sur 8 (en 1986) ou sur 4 (en 1987 et 88 ), conduisant à un fourrage plus pauvre en grain, dont la partie non-grain était directement comparable à celle de l'ensilage normal de la même variété, avec une teneur en matière sèche très proche. Les ensilages ainsi étudiés ont été en 1986 ceux des variétés Dea, Dea appauvri d'un épi sur 8 et LG 18, en 1987 ceux des variétés Dea, Dea appauvri d'un épi sur 4 et Wisconsin 416, en 1988 ceux des variétés Dea, Arcade, Arcade appauvri d'un épi sur 4 . Les variétés LG 18 et Dea sont 2 hybrides inscrits au catalogue grain avant la mise en place de la mention ensilage ; Arcade est une variété inscrite plus récemment avec la mention ensilage seulement. Ces variétés représentent des types génétiques de maïs différents dans la gamme de précocité considérée. Wisconsin 416 , hybride déjà très ancien, a été retenu parce que des essais antérieurs avaient montré qu'il avait des productivité et précocité en plante entière semblables à celles de Dea, mais avec une teneur en grain nettement plus faible (Barrière et al, 1987 ; Derieux et al, 1987). Les caractéristiques des ensilages sont données dans le tableau I à partir de prélèvements faits dans les silos. Les teneurs en grain des ensilages ont été estimées par des prélèvements de 10 échantillons de 5 plantes à la récolte. Elles sont en moyenne élevées, voisines de $50 \%$ pour les hybrides classiques, valeurs représentatives de génotypes modernes, alors que Wisconsin 416 se situe avec les hybrides appauvris en grain autour de $42 \%$. Les rendements en matière sèche des différents hybrides étaient voisins de 15 tha. Les teneurs en matière sèche des ensilages distribués se situaient au niveau de l'optimum zootechnique en 1986-87 et 1988-89, autour de $33-34 \%$, et légèrement plus faibles en 1987 88 , autour de $30 \%$. Les teneurs en azote présentent 
Tableau I. Caractéristiques des ensilages utilisés.

Essai 1986-87

Essai 1987-88

Essai 1988-89

Dea Dea LG 18

$$
\text { -1épi/8 }
$$

Dea

$\begin{array}{cc}\text { Dea } & \text { Wisconsin } \\ -1 e ́ p i / 4 & 416\end{array}$

Dea Arcade Arcade

\begin{tabular}{lrrrrrrrrr}
\hline grain (\%) & 54,0 & 51,4 & 46,1 & 47,7 & 41,4 & 41,5 & 48,6 & 48,6 & 41,6 \\
M organique (\%) & 95,2 & 93,4 & 95,5 & 95,6 & 95,4 & 95,6 & 95,6 & 96,5 & 95,8 \\
MAT (\%) & 9,5 & 9,3 & 8,9 & 7,6 & 7,6 & 7,5 & 8,2 & 7,4 & 8,3 \\
cellulose (\%) & 17,7 & 20,2 & 19,9 & 19,3 & 21,4 & 20,8 & 20,7 & 21,0 & 20,3 \\
PDIN & 59 & 57 & 55 & 47 & 47 & 46 & 50 & 45 & 51 \\
PDIE & 69 & 66 & 62 & 65 & 63 & 63 & 64 & 66 & 62 \\
\hline
\end{tabular}

une variation inter-année classique, et les valeurs de PDIN (protéines digestibles dans l'intestin permises par l'azote) et PDIE (protéines digestibles dans l'intestin permises par l'énergie), calculées à partir des mesures sur moutons standard sont très voisines pour les différents ensilages, et très proches des valeurs des tables (respectivement 52 et $66 \mathrm{~g}$ ).

Les valeurs alimentaires de ces ensilages ont été estimées par des moutons texels castrés, à l'entretien en cages à digestibilité dans les conditions standard. Ces animaux sont affouragés à volonté, de telle sorte que les refus représentent environ $10 \%$ du distribué. Chaque ensilage est distribué 2 fois par jour à 6 moutons, pendant 5 j de mesures, après une période d'accoutumance de $7 \mathrm{j}$. Les teneurs en matière organique, azote et cellulose (méthodes Kjeldall et Weende respectivement) ont été mesurées sur un échantillon moyen des 6 moutons et des $5 \mathrm{j}$ de mesures. Dans quelques cas, 1 ou 2 moutons ont classiquement été éliminés en raison de leur comportement anormal (quantités ingérées trop faibles en particulier). Les digestibilités ou coefficients d'utilisation digestive (CUD) de la matière organique et de la cellulose sont estimés en moyenne des moutons retenus pour les $5 \mathrm{j}$ de mesure. Les mesures ont été réalisées pour chacun des ensilages par un (en 1986-87) et deux (en $1987-88,1988-89$ ) lots de moutons. De plus, en 198889 , les mesures ont également été répétées pour chacun des ensilages avec 2 lots de moutons affouragés en quantités limitées, correspondant aux besoins d'entretien ( $40 \mathrm{~g}$ de fourrage par kilo de poids métabolique).

Les mesures de digestibilité in vitro (méthode de Tilley, Terry, 1963, et méthode enzymatique de Lila et al, 1984) ont été effectuées sur les ensilages, à partir d'échantillons prélevés lors de l'affouragement des moutons standard.

Le troupeau expérimental de 55 vaches laitières FFPN Holstein d'un niveau moyen de production de $7500 \mathrm{~kg}$ de lait environ a permis de constituer 3 lots comparables de 8 animaux en 1986-87, dont 2 primipares, et 10 animaux en 1987-88 et 1988-89, dont respectivement 2 et 4 primipares. Excepté un animal dans chaque lot en 1986-87 et 1987-88, les vêlages sont des vêlages d'automne. Les études sont conduites dans la période qui suit le pic de lactation.
Outre la date de vêlage et le rang de la lactation, les critères d'allotement retenus ont été le niveau de production laitière, le poids, les taux protéiques et butyreux mesurés au cours d'une période pré-expérimentale de 2 (en 1986-87 et 1988-89) ou 3 (en 1987-88) semaines. L'homogénéité des lots s'est avérée satisfaisante, même si une certaine dérive a été observée au cours de la pré-expérience qui a suivi la mise en lot, pour les productions de lait en 1986-87 et 1987-88, et même si l'allotement n'était pas parfait pour les taux butyreux en 1988-89 (tableau II), facteurs qui seront corrigés par analyse de covariance. La période expérimentale a duré 11 semaines durant l'hiver 1986-87, mais les données de la seconde semaine ont été éliminées (ensilages gelés); elle a duré 12 semaines durant l'hiver 1987-88 et 14 semaines en 1988-89.

Les ensilages de maïs bien conservés ont été distribués à volonté (5 à $10 \%$ de refus), avec un suivi individuel des quantités ingérées au moyen de portillons électromagnétiques. Cette ration de base a été équilibrée de façon identique pour les 3 ensilages par l'apport à l'auge de tourteaux tannés de soja-colza (1,3 à $1,4 \mathrm{~kg}$ ), d'urée (130 à $230 \mathrm{~g}$ ) et minéraux de type $12-16-5$ (200 à $250 \mathrm{~g}$ ), permettant la production de 22 (ou 17) kg de lait par les multipares (ou les primipares). L'apport de concentré de production a été réalisé individuellement en salle de traite selon un schéma prédéterminé avec un concentré adapté, de type soja-céréale, fermier ou industriel ( 120 à $130 \mathrm{~g}$ de PDI par UFL), à raison de $1 \mathrm{~kg}$ pour $3 \mathrm{~kg}$ de lait au-dessus des niveaux supposés couverts par la ration de base corrigée. La diminution théorique de la production de lait prise en compte était classiquement de 2,0 et 2,5\% par semaine pour respectivement les primipares et multipares (Hoden et al, 1988).

Les mesures ont été effectuées individuellement 4 j par semaine pour les quantités ingérées, les teneurs en matière sèche du distribué et des refus, et $2 \mathrm{j}$ non consécutifs par semaine, soit 4 traites, pour les taux protéiques et butyreux; les productions laitières ont été enregistrées 4 j par semaine en 1986-87 et quotidiennement en 1987-88 et 1988-89 par des compteurs à lait.

Toutes les teneurs en matière sèche des ensilages ont été corrigées de la perte des produits volatils durant l'étuvage en utilisant une régression non linéai- 
Tableau II. Caractéristiques des vaches laitières mises en expérimentation.

\section{Mise en lot}

\begin{tabular}{|c|c|c|c|}
\hline Essai 1986-87 & Lot 1 & Lot 2 & Lot 3 \\
\hline $\begin{array}{l}\text { Poids vif }(\mathrm{kg}) \\
\text { Production laitière }(\mathrm{kg}) \\
\text { Taux butyreux }(\mathrm{g} \% \mathrm{o}) \\
\text { Taux protéique }(\mathrm{g} \% \mathrm{o}) \\
\text { Stade de lactation } \\
\text { en début }\end{array}$ & $\begin{array}{l}583 \\
29,8 \\
41,4 \\
29,3\end{array}$ & $\begin{array}{l}582 \\
29,4 \\
43,6 \\
29,3\end{array}$ & $\begin{array}{l}582 \\
29,3 \\
42,9 \\
29,4\end{array}$ \\
\hline d'expérimentation (jours) & 98 & 103 & 119 \\
\hline
\end{tabular}

Essai 1987-88

Moyenne de 4 semaines de pré-expérimentation après la mise en lot

\section{Poids vif $(\mathrm{kg})$}

606

610

615

Production laitière $(\mathrm{kg})$

26,1

23,7

25,1

Taux butyreux ( $\mathrm{g} \%$ )

44,2

44,2

43,4

Taux protéique $(\mathbf{g} \%)$

30,6

31,8

30,1

Stade de lactation

en début

d'expérimentation (jours)

119

129
Moyenne de 2 semaines de pré-expérimentation après la mise en lot
Poids vif $(\mathrm{kg})$

Production laitière $(\mathrm{kg})$

Taux butyreux ( $\mathrm{g} \%$ o)

Taux protéique ( $\mathrm{g} \%$ )

Stade de lactation

en début

d'expérimentation (jours)

\section{6}

29,6

43,9

31,3

649

29,5

41,5

30,4

86
634

29,4

46,0

30,9

re construite à partir des travaux de Dulphy et al $(1975,81)$ et Jarrige (1988).

Les variations de poids des animaux au cours de l'expérimentation ont été estimées par une pesée faite en début et en fin d'expérimentation $2 \mathrm{j}$ consécutifs. Un suivi par une simple pesée a été réalisé toutes les 3 semaines. Ces variations de poids vifs ont été corrigées des variations de poids des contenus digestifs des animaux (Chilliard et al, 1987). La très forte variabilité individuelle pour ce caractère et le faible nombre de degrés de liberté ne permettent que difficilement de mettre en évidence d'éventuelles différences significatives. Par ailleurs, en raison d'une fréquence anormale de données aberrantes, les valeurs de la pesée de fin d'expérimentation de l'essai de 1987-88 ont été estimées par extrapolation des valeurs des pesées précédentes.

Les bilans énergétiques ont été réalisés par différence entre les besoins des animaux (entretien, production de lait, variation de poids corrigées) et les apports de la ration en prenant comme valeur énergétique UFL de référence pour l'ensilage de maïs celle

obtenue à partir de la mesure sur moutons standards, et en tenant compte des interactions négatives entre fourrages et concentrés liées à l'utilisation de l'énergie (Faverdin et al, 1987). Les valeurs énergétiques UFL auxquelles ont été valorisés chacun des ensilages de maïs par les vaches laitières ont été estimées par les valeurs qui annulent ces bilans entre apports et besoins.

Les analyses de variance multivariables (programme anvarm, Bachacou et al, 1981) ont été réalisées en prenant en compte un effet type d'ensilage de maïs distribué, un effet animal correspondant à 3 animaux homogènes sur les caractéristiques de mise en lot et répartis chacun dans un lot, un effet semaine d'expérimentation, et les interactions type d'ensilage semaine d'expérimentation et type d'ensilage animal. Avec un tel protocole comprenant des observations répétées, le rapport des carrés moyens type d'ensilage sur le carré moyen résiduel peut ne pas être distribué comme une variable $F$, avec donc un risque de considérer comme significatives des différences qui ne le sont pas (Little, Hills, 1978). Un des tests $F$ possibles consiste alors à rapporter la variation interlot à la variation intralot, et donc, de manière analogue à un dispositif split-plot, de faire le rapport des carrés moyens type d'ensilage sur l'interaction type d'ensilage animal. Les valeurs expérimentales ont été ajustées des covariables significatives au cours de la période de pré-expérimentation (production de lait brut en 1986-87 et 1987-88 et taux butyreux en 1988-89). Les études n'ont été faites que pour les lots complets d'animaux, des récapitulations préliminaires n'ayant pas montré de différences de comportement entre vaches primipares et multipares. Les moyennes présentées sont ajustées des effets du modèle.

\section{RÉSULTATS ET DISCUSSIONS}

\section{Variabilité mesurée par les vaches laitières}

Les résultats obtenus sur vaches laitières sont présentés dans les tableaux III, IV et V, pour chacune des 3 années d'expérimentation.

En 1986-87, les quantités de matière sèche de maïs ingérées par les animaux ne sont pas significativement différentes pour les 3 ensilages. L'ingestion apparemment un peu plus élevée de LG 18 peut être en partie liée a sa teneur en matière sèche légèrement plus élevée. Au contraire, la plus faible teneur en matière sèche de l'ensilage de Dea appauvri en épi a été compensée par une plus forte ingestion de matière fraîche. Les productions laitières sont similaires pour les 3 ensilages en lait brut, ou en lait à $4 \%$. De même, les quantités de matières grasses ou de matières protéiques produites sont semblables avec les différents ensilages. Les variations de poids observées entre les différents lots sont très faibles. Les bilans énergétiques sont inégalement déficitaires quand ils sont estimés sur les valeurs UFL des ensilages de maïs obtenues 
Tableau III. Valorisation des 3 ensilages par les vaches laitières. Essai 1986-87. F maïs $=$ Dea, Dea -1 épi/8, LG $18 ; F$ à 2 et 14 ddl ; ns = non significatif.

\begin{tabular}{lcccc}
\hline $\begin{array}{l}\text { Essai 1986-87 } \\
\text { 77 } j\end{array}$ & Dea & $\begin{array}{c}\text { Dea } \\
-1 e ́ p i / 8\end{array}$ & LG 18 & F maïs \\
\hline Ingéré maïs (kg) & 15,6 & 15,6 & 16,5 & $1,27 \mathrm{~ns}$ \\
M sèche du distribué (\%) & 34,1 & 33,0 & 35,4 & - \\
Ration totale (kg) & 19,2 & 19,1 & 20,1 & $1,10 \mathrm{~ns}$ \\
Lait brut (kg) & 23,2 & 23,2 & 22,8 & $0,05 \mathrm{~ns}$ \\
Lait à 4\% (kg) & 24,4 & 24,6 & 24,0 & $0,11 \mathrm{~ns}$ \\
Matières grasses (g) & 1010 & 1017 & 990 & $0,11 \mathrm{~ns}$ \\
Matières protéiques (g) & 702 & 713 & 722 & $0,09 \mathrm{~ns}$ \\
Taux butyreux (g\%o) & 44,8 & 43,7 & 32,9 & $0,33 \mathrm{~ns}$ \\
Taux protéique (g\%) & 31,1 & 30,8 & 26,8 & - \\
Variation de poids (kg) & 20,3 & 12,2 & & - \\
corrigée ingestion & & & $-1,71$ & - \\
Bilan énergétique (UFL) & $-0,75$ & $-1,33$ & & \\
sur valeurs UFL moutons & & & 0,89 & \\
Valorisation énergétique (UFL) & 0,93 & 0,91 & & \\
par les vaches laitières & & &
\end{tabular}

avec les moutons standard. La valorisation énergétique des ensilages de Dea apparaît similaire à 0,93 et 0,91 UFL, celle de LG 18 valant 0,89 UFL, valeurs classiques attendues pour de bons ensilages de maïs.

En 1987-88, les quantités de matières sèches ingérées de chacun des 3 ensilages sont semblables. Les productions laitières permises par les ensilages de Dea normal ou appauvri en épis sont identiques, en lait brut ou en lait à $4 \%$. En revanche, l'ensilage de Wisconsin 416 permet une production laitière journalière supérieure de $1,5 \mathrm{~kg}$ de lait brut (différence significative), ou de $1,2 \mathrm{~kg}$ de lait à $4 \%$ à celle des 2 autres ensilages (différence non significative, mais qui peut en partie résulter de la grande variabilité inter-animaux pour les taux butyreux). Les taux butyreux et protéiques du lait produit avec Wisconsin 416 sont non significativement inférieurs à ceux des laits produits avec les 2 autres ensilages. Les variations de poids sont peu différentes pour chacun des lots. Le bilan énergétique, estimé à partir des valeurs UFL obtenues sur les moutons standard, est légèrement excédentaire pour les animaux nourris à l'ensilage de Dea, déficitaire mais proche de l'équilibre avec l'ensilage de Dea appauvri en épis, et plus nettement déficitaire pour Wisconsin 416. Les 2 ensilages de Dea semblent valorisés par les vaches laitières de façon très semblable à 0,91 et 0,88 UFL, alors que, à 0,96 UFL, la valeur énergétique de Wisconsin 416 apparaît supérieure.

En 1988-89, les 3 ensilages sont ingérés de façon similaire par les animaux, malgré une teneur en matière sèche un peu plus élevée de l'ensilage de Dea. Les productions de lait brut ou de lait à $4 \%$ permises par les 3 ensilages ne sont pas significativement différentes. Les quantités de matières grasses et protéiques produites, de même que les taux butyreux et protéiques sont semblables pour les 3 ensilages. Les reprises de poids sont du même ordre pour chacun des ensilages, y compris pour l'ensilage appauvri en épis. Les bilans énergétiques montrent de nets déficits quand ils sont estimés à partir des valeurs UFL données par les moutons standard. Les valorisations énergétiques des ensilages par les vaches laitières apparaissent très élevées et proches, entre 1,00 et 1,04 UFL, avec de nouveau une très bonne valorisation de l'ensilage appauvri en épis. Ces valeurs très élevées peuvent s'expliquer à la fois par les très bonnes conditions de végétations du maïs, par la mise en essais d'animaux moins avancés dans leurs lactations et par un suivi très rigoureux des complémentations.

Pour chacune de ces 3 années, les interactions entre les ensilages de maĩs distribué et la semaine d'expérimentation sont très généralement non significatives pour les variables liées à la production laitière, alors qu'elles le sont pour les quantités de maïs ingérées en raison essentiellement des variations hebdomadaires de teneurs en matière sèche des ensilages.

II est possible de discuter l'ensemble de ces résultats en l'absence de biais éventuels liés à une suralimentation des animaux. En effet les bilans énergétiques, estimés selon les normes les plus récentes de l'alimentation des vaches 
Tableau IV. Valorisation des 3 ensilages par les vaches laitières. Essai 1987-88.

$\mathrm{F}$ maïs $=$ Dea, Dea -1 épi $/ 4$, Wisconsin $416 ; \mathrm{F}$ à 2 et $18 \mathrm{ddl} ; \mathrm{ns}=$ non significatif $;{ }^{\star *}=$ significatif $p \geq 0,99$.

\begin{tabular}{|c|c|c|c|c|}
\hline $\begin{array}{l}\text { Essai 1987-88 } \\
\qquad 84 j\end{array}$ & Dea & $\begin{array}{l}\text { Dea } \\
\text {-1épi/4 }\end{array}$ & $\begin{array}{l}\text { Wisconsin } \\
\quad 416\end{array}$ & $F$ mä̈s \\
\hline $\begin{array}{l}\text { Ingéré maïs }(\mathrm{kg}) \\
\text { M sèche du distribué }(\%) \\
\text { Ration totale }(\mathrm{kg}) \\
\text { Lait brut }(\mathrm{kg}) \\
\text { Lait à } 4 \%(\mathrm{~kg}) \\
\text { Matières grasses }(\mathrm{g}) \\
\text { Matières protéiques }(\mathrm{g}) \\
\text { Taux butyreux }(\mathrm{g} \% \mathrm{~g}) \\
\text { Taux protéique }(\mathrm{g} \%)\end{array}$ & $\begin{array}{c}16,4 \\
29,9 \\
18,5 \\
20,3 \\
21,8 \\
912 \\
648 \\
45,1 \\
32,3\end{array}$ & $\begin{array}{c}16,4 \\
29,6 \\
18,5 \\
20,4 \\
21,9 \\
916 \\
651 \\
45,0 \\
32,2\end{array}$ & $\begin{array}{c}16,1 \\
30,5 \\
18,2 \\
21,8 \\
23,0 \\
953 \\
683 \\
43,6 \\
31,5\end{array}$ & $\begin{array}{l}0,40 \mathrm{~ns} \\
\overline{0} \\
0,48 \mathrm{~ns} \\
7,066^{\star *} \\
2,33 \mathrm{~ns} \\
0,69 \mathrm{~ns} \\
2,42 \mathrm{~ns} \\
0,35 \mathrm{~ns} \\
0,43 \mathrm{~ns}\end{array}$ \\
\hline $\begin{array}{l}\text { Variation de poids (kg) } \\
\text { corrigée ingestion }\end{array}$ & 25,1 & 16,7 & 22,6 & - \\
\hline $\begin{array}{l}\text { Bilan énergétique (UFL) } \\
\text { sur valeurs UFL moutons }\end{array}$ & 0,52 & $-0,35$ & $-1,25$ & - \\
\hline $\begin{array}{l}\text { Valorisation énergétique (UFL) } \\
\text { par les vaches laitières }\end{array}$ & 0,91 & 0,88 & 0,96 & - \\
\hline
\end{tabular}

Tableau V. Valorisation des 3 ensilages par les vaches laitières. Essai 1988-89.

$\mathrm{F}$ maïs = Dea, Arcade, Arcade -1épi $/ 4 ; \mathrm{F}$ à 2 et 18 ddl ; ns = non significatif.

\begin{tabular}{|c|c|c|c|c|}
\hline $\begin{array}{c}\text { Essai 1988-89 } \\
112 j\end{array}$ & Dea & Arcade & $\begin{array}{l}\text { Arcade } \\
-1 \text { épi/4 }\end{array}$ & F maïs \\
\hline Ingéré maïs $(\mathrm{kg})$ & 15,1 & 16,1 & 15,7 & $2,28 \mathrm{~ns}$ \\
\hline $\begin{array}{l}\text { M sèche du distribué }(\%) \\
\text { Ration totale }(\mathrm{kg})\end{array}$ & $\begin{array}{l}34,9 \\
18,1\end{array}$ & $\begin{array}{l}32,6 \\
19,0\end{array}$ & $\begin{array}{l}32,0 \\
18,7\end{array}$ & $\overline{1}, 40 \mathrm{~ns}$ \\
\hline Lait brut $(\mathrm{kg})$ & 24,9 & 25,1 & 24,9 & $0,02 \mathrm{~ns}$ \\
\hline Lait à $4 \%(\mathrm{~kg})$ & 25,7 & 26,3 & 26,2 & $0,01 \mathrm{~ns}$ \\
\hline Matières grasses (g) & 1051 & 1087 & 1084 & $0,19 \mathrm{~ns}$ \\
\hline Matières protéiques (g) & 776 & 770 & 786 & $0,10 \mathrm{~ns}$ \\
\hline Taux butyreux $(\mathrm{g} \% \mathrm{c})$ & 42,6 & 43,3 & 43,4 & $0,18 \mathrm{~ns}$ \\
\hline Taux protéique $(\mathrm{g} \%$ ) & 31,7 & 30,8 & 31,7 & $0,54 \mathrm{~ns}$ \\
\hline $\begin{array}{l}\text { Variation de poids }(\mathrm{kg}) \\
\text { corrigée ingestion }\end{array}$ & 28,3 & 33,7 & 32,1 & - \\
\hline $\begin{array}{l}\text { Bilan énergétique (UFL) } \\
\text { sur valeurs UFL moutons }\end{array}$ & $-3,17$ & $-1,62$ & $-3,34$ & - \\
\hline $\begin{array}{l}\text { Valorisation énergétique (UFL) } \\
\text { par les vaches laitières }\end{array}$ & 1,04 & 1,00 & 1,04 & - \\
\hline
\end{tabular}

laitières (Jarrige, 1988) et en prenant pour les ensilages de maïs la valeur énergétique de référence fournie par les mesures sur moutons standard, sont négatifs ou nuls, si ce n'est une valeur légèrement positive pour l'ensilage de Dea en 1987-88. Par ailleurs les bilans azotés sont similaires, chaque année, pour chacun des hybrides, équilibrés ou très légèrement excédentaires, et d'autant moins excédentaires que la production de lait à $4 \%$ est plus élevée.
La comparaison, durant ces 3 années, d'ensilages normaux ou appauvris en épis de Dea ou Arcade montre qu'il n'y a pas d'effet direct de la teneur en grain, à niveau élevé, entre 51,4 et $54,0 \%$, ou entre 41,5 et $48,6 \%$, ni sur la production laitière, ni sans doute sur les reprises de poids des animaux. II n'y en a donc pas non plus sur la valorisation énergétique des ensilages, comme semblent d'ailleurs le confirmer les bilans énergétiques. Puisque d'autre part les quantités 
de matière sèche d'ensilage ingérées étaient semblables, ces résultats montrent que, même avec des rations relativement pauvres en concentrés (entre 9 et $18 \%$ ), une réduction de la teneur en grain de l'ensilage conduit à une meilleure valorisation de la partie non-grain, ou à une meilleure valorisation de l'ensemble de la ration. En l'absence de compensations de ce type, une réduction de $7 \%$ des teneurs en grain, avec des quantités égales d'ensilage ingérées, conduirait à une diminution de la production laitière résultant d'une diminution des apports énergétiques autour de la satisfaction des besoins voisine de $0,8 \mathrm{~kg}$ de lait par animal et par jour, avec une mobilisation des réserves de l'ordre de $30 \mathrm{~kg}$ sur l'ensemble de la période expérimentale (Faverdin et al, 1987).

Ces observations rejoignent celles de ElShazy et al (1961) et de Henning et al (1980) qui ont mis en évidence les antagonismes entre les flores amilolytique et cellulolytique du rumen. Et ces derniers ont de plus fait l'hypothèse d'un effet inhibiteur direct de l'amidon ou des sucres qui en dérivent sur la synthèse ou l'activité des cellulases ou des hémicellulases. Joanning et al (1981), confirmant les observations de Byers (1974), ont par ailleurs montré une réduction de la digestibilité à la fois de l'amidon et des fibres dans des rations mixtes ensilage de maïs grains de maiis aplatis, comparées à des rations d'ensilage seul, mais ces expérimentations étaient conduites sur bouvillons. II n'est pas possible de conclure ici soit à une réduction de la digestibilité des fibres, soit à une réduction conjointe de la digestibilité des fibres et de l'amidon dans les rations normalement pourvues en grain, mais il semble y avoir au moins une certaine équivalence entre amidon et cellulose, dans ces proportions, dans ce type de ration et pour les vaches laitières, puisque la diminution de la teneur en grain ne semble modifier ni la production de lait, ni la valorisation apparente des ensilages de maïs ingéré.

Les effets de la variabilité génétique sur la production journalière de lait à $4 \%$ sont de l'ordre de $0,5 \mathrm{~kg}$ de lait, mais non significatifs, entre LG 18 et Dea ou entre Dea et Arcade, variétés modernes non apparentées. En revanche, l'écart de production journalière de lait brut permise par l'ensilage est de $1,5 \mathrm{~kg}$ entre Dea et Wisconsin 416. Ces premiers résultats, même limités, mettent en évidence l'existence possible d'un effet du génotype de maiis ensilé sur les performances de vaches laitières à haut potentiel, sans que l'on puisse conclure en fait à un effet lié seulement à une variabilité pour la digestibilité ou également à la valeur énergétique. Le protocole expérimental utilisé permet toutefois d'exclure des effets d'ap- ports d'azote différents. Avec la gamme de génotypes étudiée, il ne semble pas non plus apparaître de différences ou d'effets liés à des quantités d'ensilage ingérées différentes. Ces résultats montrent aussi que les sources de variabilité les plus intéressantes pour le maïs ensilage ne sont peut-être pas classiquement présentes dans la gamme de matériel actuellement utilisée, qui est globalement issue de 40 ou 50 ans de sélection grain. II faudra sans doute développer des ressources génétiques spécifiques pour obtenir des progrès notables en terme de production laitière permise par une quantité donnée de matière sèche d'ensilage de maïs.

La comparaison des ensilages de Dea et Wisconsin $\mathbf{4 1 6}$ montre que des teneurs en cellulose proches peuvent conduire à des résultats zootechniques différents. Pour des génotypes d'un niveau de précocité donné, plus que la teneur en cellulose, et même plus que la teneur en lignine, c'est la proportion de tissus lignifiés dans la plante et la composition monomérique de la lignine qui sont les facteurs clés de la digestibilité des plantes entières de maîs (Grand et al, 1985 ; Grenet, Barry, 1988 ; Jung, 1989 ; Grenet, Barry, com pers). Ainsi les parois dont la lignine est riche en groupements syringiles peuvent être en partie dégradées dans le rumen, alors que les parois dont la lignine est riche en groupements cinnamaldéhydes ne sont pas dégradées.

\section{Prédiction de la valeur énergétique}

Le tableau VI récapitule les différentes estimations, prédictions ou mesures de valeur alimentaire des ensilages consommés par les vaches laitières. La valeur alimentaire de référence estimée par les moutons standard distingue nettement les ensilages normaux de leurs isogéniques appauvris en grains avec une différence de 2 à 4 points pour les CUD de matière organique, et de 0,07 ou 0,08 UFL $/ \mathrm{kg}$ pour la valeur énergétique, sachant que la précision classique de cette estimation est de $\pm 0,02$ UFL $\mathrm{kg}$. Mais ces différences ne se retrouvent pas sur vaches laitières. En terme de valorisation énergétique par les vaches laitières, le classement des ensilages est correctement prédit entre Dea et LG 18, mais inversé entre Dea et Arcade et surtout entre Dea et Wisconsin 416. En revanche, le meilleur CUD de la cellulose de Wisconsin 416 est bien mis en évidence par les moutons standard, la valeur plus faible du CUD de la cellulose de l'ensilage d'Arcade appauvri d'un épi sur 4 est difficilement explicable. D'une manière générale, il semblerait que l'outil mouton standard soit très sensible aux teneurs en grain, et moins apte à 
Tableau VI. Valeur alimentaire des 3 ensilages mesurée par les moutons standard et prédictions par différentes méthodes.

Essai 1986-87 Dea $\begin{gathered}\text { Dea } \\ \text {-1épi/8 }\end{gathered}$ LG 18

\begin{tabular}{lccc} 
CUD matière organique (\%) & 69,5 & 67,5 & 64,3 \\
CUD cellulose (\%) & 50,9 & 53,5 & 48,4 \\
UFL moutons & 0,88 & 0,82 & 0,79 \\
UFL vaches laitières & 0,93 & 0,91 & 0,89 \\
Digestibilité (Tilley, Terry) & 68,7 & 71,1 & 67,5 \\
Digestibilité enzymatique & & & \\
(APC) & 87,4 & 88,2 & 87,7 \\
UFL cellulose ( $\pm 0,03)$ & 0,91 & 0,86 & 0,88 \\
UFL grain $( \pm 0,03)$ & 0,96 & 0,94 & 0,92 \\
\hline
\end{tabular}

\begin{tabular}{lcc}
\hline Essai 1987-88 Dea $\begin{array}{c}\text { Dea } \\
\text {-1épi/4 }\end{array}$ & $\begin{array}{c}\text { Wiscon- } \\
\text { sin416 }\end{array}$ \\
\hline
\end{tabular}

$\begin{array}{lccc}\text { CUD matière organique (\%) } & 72,6 & 68,4 & 68,8 \\ \text { CUD cellulose (\%) } & 49,1 & 48,0 & 53,9 \\ \text { UFL moutons } & 0,94 & 0,86 & 0,88 \\ \text { UFL vaches laitières } & 0,91 & 0,88 & 0,96 \\ \text { Digestibilité (Tilley, Terry) } & 65,7 & 65,5 & 62,3 \\ \text { Digestibilité enzymatique } & & & \\ \text { (APC) } & 88,0 & 85,4 & 86,6 \\ \text { UFL cellulose ( } \pm 0,03) & 0,88 & 0,85 & 0,86 \\ \text { UFL grain }( \pm 0,03) & 0,93 & 0,90 & 0,90\end{array}$

\begin{tabular}{rr} 
Essai 1988-89 Dea Arcade $\begin{array}{r}\text { Arcade } \\
\text {-1épi/4 }\end{array}$ \\
\hline
\end{tabular}

\begin{tabular}{lccc} 
CUD matière organique (\%) & 66,3 & 69,5 & 66,0 \\
CUD cellulose (\%) & 51,5 & 51,2 & 44,3 \\
UFL moutons & 0,83 & 0,90 & 0,83 \\
UFL vaches laitières & 1,04 & 1,00 & 1,04 \\
Digestibilité (Tilley, Terry) & 60,2 & 59,7 & 58,4 \\
Digestibilité enzymatique & & & \\
(APC) & 78,1 & 75,8 & 76,9 \\
UFL cellulose $( \pm 0,03)$ & 0,86 & 0,86 & 0,87 \\
UFL grain $( \pm 0,03)$ & 0,93 & 0,93 & 0,90 \\
\hline
\end{tabular}

valoriser une cellulose de bonne qualité que les vaches laitières, même lorsque le CUD de la cellulose qu'il mesure confirme une meilleure digestibilité de ce constituant. Les valeurs énergétiques des ensilages prédites par les moutons standard sont d'ailleurs presque systématiquement inférieures à celles auxquelles sont valorisés ces mêmes ensilages par les vaches laitières. Les mesures en quantités limitées de fourrage, (effectuées en 1988-89), conduisent à une diminution de la variabilité observée (respectivement $70,4,72,0,71,6 \%$ pour les CUD de la matière organique de Dea, Arcade et Arcade appauvri, valeurs non significativement différentes). Ces résultats confirment, en accord avec
Aerts (1986), la difficulté de la prédiction de la valeur énergétique d'un fourrage dans une ration pour des gros ruminants alimentés à 3 fois le niveau de la maintenance, à partir de mesures sur moutons à l'entretien avec ce fourrage en plat unique, dans le cas d'observations de variations intraspécifiques d'amplitude limitée.

Les mesures de digestibilité in vitro faites avec du jus de rumen (méthode de Tilley, Terry, 1963), ont tendance à sous-estimer la digestibilité des ensilages de maïs, alors que les solubilités enzymatiques conduiraient plutôt à une surestimation. En revanche ces 2 méthodes ne distinguent pas les hybrides normaux des mêmes hybrides appauvris en grain. Elles semblent aussi conduire à un certain tassement de la variabilité mise en évidence par les moutons standard, mais c'est aussi cela qui est observé sur vaches laitières hormis la relative supériorité de Wisconsin 416 comparé à Dea, qui n'est pas mise en évidence par ces méthodes in vitro. Toutefois les différences à mettre en évidence sont probablement proches de l'ordre de grandeur des erreurs résiduelles des méthodes in vitro employées, en particulier pour la méthode de Tilley et Terry. Une autre méthode de solubilité enzymatique, utilisée par Biston et Dardenne (1988), semble avoir une précision analytique supérieure à celle que nous avons employée. Mais ces méthodes in vitro représentent dans tous les cas un compromis entre une valorisation effective d'un ensilage dans une ration par un gros ruminant, et une approche de celle-ci soit par une digestion par des micro-organismes plus ou moins représentatifs dans un milieu clos, soit par une digestion enzymatique en principe totale des constituants biologiquement dégradables.

Enfin, il faut noter des effets année importants, ce qui est relativement classique, les travaux de Deinum (1987) ayant toutefois montré une stabilité des classements des génotypes pour les critères de digestibilité entre années et entre lieux. De plus, les différences interannuelles de valorisation des ensilages par les vaches laitières sont exacerbées par les durées un peu différentes des essais.

Les régressions sur la teneur en cellulose ou la teneur en grain (Andrieu, 1984) ont été établies pour permettre une estimation rapide de la valeur énergétique des ensilages, en l'absence de mesures de digestibilité difficiles à réaliser, et avant la diffusion des prédictions à partir des réflectances dans le proche infrarouge. Ces équations ont été établies sur des gammes larges de variations de teneurs en grain ou en cellulose, avec des références obtenues sur des moutons standard. Elles sont de bons outils de terrain, mais ne permettent évidemment pas la 
prise en compte des variations observées ici sur vaches laitières. De plus, pour la prédiction de la valeur énergétique à partir des teneurs en grain, il semble en fait exister plusieurs régressions différentes dans les différents intervalles de teneur en grain.

\section{CONCLUSIONS}

Ces expérimentations, qui pour des raisons évidentes n'ont pu être réalisées que sur un petit nombre de génotypes, et qu'il ne faut sans doute pas généraliser de façon hâtive, laissent penser qu'une variabilité génétique du maiis peut avoir des conséquences directes sur les productions des vaches laitières consommant les différents ensilages, même si les hybrides modernes testés apparaissent valorisés de manière très similaire. Elles montrent aussi clairement qu'il est possible de perdre presque 10 points de teneur en grain dans l'ensilage sans perdre en valeur énergétique, au moins avec les hybrides testés, en bonnes conditions de végétation, et avec la complémentation utilisée. Or des travaux de sélection montrent qu'il est possible de progresser nettement en productivité en plante entière si l'on accepte de perdre un peu en productivité en grain, en choisissant des hybrides dont le fonctionnement physiologique est orienté vers la production de biomasse, avec une durée de surface foliaire (LAD) élevée et une maturation rapide, plutôt que vers des transferts intenses vers l'épi. Et cela semble même possible avec des combinaisons originales de lignées appartenant à un fond génétique issu de près d'un demi-siècle de sélection pour la productivité en grain. Toutefois Struik (1983) a mis en évidence une tendance nette vers une réduction de la digestibilité in vitro des hybrides inscrits au catalogue hollandais entre 1969 et 1983, résultats qui semblent confirmés avec des hybrides présentés ultérieurement à cette étude. Deinum et Struik (1985) ont ainsi pu démontrer que des grains de productivité en biomasse pouvaient être complètement ou partiellement annulés au niveau de la biomasse digestible. Ces effets sont probablement dus, pour la base génétique étudiée, à l'évolution du matériel et en particulier à l'abandon de certaines lignées, ainsi qu'à des efforts intenses de sélection pour la résistance aux verses, sans suivi simultané de la valeur alimentaire. L'hybride Wisconsin 416 , dont la digestibilité de la cellulose et la valeur énergétique sont relativement plus élevées, se caractérisait aussi par sa beaucoup plus grande sensibilité à la verse en végétation. Les travaux de Derieux et al (1987) avaient aussi montré sa sensibilité aux pourritures des tiges, plus grande que celle des hybrides du type Dea. S'il n'est pas nécessaire que les hybrides destinés à l'ensilage aient un niveau très élevé de résistance aux pourritures des tiges, en particulier pour les aspects de résistance mécanique des tiges à surmaturité, en revanche ces hybrides devront être très résistants à la verse en végétation. Mais la qualité de l'ancrage racinaire est sans doute une composante majeure de la résistance à la verse en végétation, caractère qui, sous l'aspect niveau d'insertion des racines ou géométrie du système racinaire, doit être relativement indépendant de la teneur en lignine, de sa composition et des maillages lignines-celluloses, et qui pourrait alors être sélectionné simultanément à une meilleure digestibilité des plantes.

Ces résultats confirment l'intérêt de programmes spécifiques de sélection de maïs destinés à une utilisation sous forme d'ensilage, incluant des tests de digestibilité. Ils mettent clairement en évidence la nécessité de développer des pools génétiques originaux, que seuls des progrès nets seront statistiquement significatifs en raison de la variabilité de l'outil de test zootechnique. Ce sont aussi ces approches qui permettront d'infléchir la tendance actuelle à la baisse de la digestibilité des hybrides présentés à l'inscription (Deinum, Struik, 1985). Mais ces travaux de sélection ne seront efficaces que si les obtenteurs disposent d'un test de digestibilité in vitro facile à mettre en œuvre sur de grandes séries, peu coûteux et surtout bon prédicteur de la variabilité observée sur vaches laitières. Dans une première étape de la sélection, on peut penser qu'une méthode de digestibilité enzymatique, calibrée en réflectance dans le proche infrarouge (NIRS) à partir de nombreux essais multiannuels et multilocaux, permettra de trier parmi les génotypes productifs et résistants à la verse, ceux dont la digestibilité in vitro est élevée. Les travaux de Biston et Dardenne (1988) montrent d'ailleurs la fiabilité des prédictions en NIRS. Mais les méthodes enzymatiques de référence devront peut-être être affinées pour que ces valeurs de digestibilités élevées donnent des classements et des écarts entre génotypes les plus proches possibles des valeurs observées sur vaches laitières. Ainsi que le proposent Deinum et Struik (1989), il se pourrait que la digestibilité des parois soit un meilleur prédicteur de la digestibilité effective des hybrides que la digestibilité de l'ensemble de la matière sèche, mais cette mesure devra acquérir un niveau de répétabilité suffisant pour être étalonnée dans de bonnes conditions sur infra-analyser. On peut aussi envisager d'étalonner des digestibilités in sacco, même si dans ce cas les analyses de 
référence sont plus difficiles à réaliser. II faudra par ailleurs acquérir une meilleure compréhension des composantes de la digestibilité du maïs dans une ration chez des vaches laitières à haut potentiel ainsi qu'une meilleure connaissance de la lignification du maïs en particulier pour ce qui concerne l'organisation structurelle des tissus et la variabilité des maillages entre lignines et celluloses. II faudra également mettre en place des essais comparatifs sur vaches laitières avec des génotypes de maïs préalablement étudiés et triés pour cet ensemble de caractères, et en particulier avec des génotypes apparaissant nettement dissemblables pour leur digestibilité estimée par les différentes méthodes in vitro.

\section{REMERCIEMENTS}

Nous remercions $A$ Hoden, $P$ Faverdin (INRA, Station de recherches sur la vache laitière) et $Y$ Hébert (INRA, SAPF, Lusignan) pour les conseils donnés lors de l'élaboration des protocoles, du suivi zootechnique ou de la rédaction de ces essais. Nous remercions $E$ Grenet (INRA, Station de recherches sur la nutrition des herbivores) pour les informations et les publications communiquées.

\section{RÉFÉRENCES}

Aerts JV (1986) Use of sheep as pilot animals for predicting digestibility of feeds to be fed to dairy cows. IDF Bulletin 196, 22-24

Andrieu J (1984) Prévision de la digestibilité et de la valeur énergétique des ensilages de maïs à partir de la composition de la plante à la mise en silo. Bull Tech CRZV Theix 56, 5-8

Andrieu J, Demarquilly C (1974) Valeur alimentaire du maïs fourrage. II. Influence du stade de végétation, de la variété, du peuplement, de l'enrichissement en épis et de l'addition d'urée sur la digestibilité et l'ingestibilité de l'ensilage de maïs. Ann Zootech 23, 1-25

Bachacou J, Masson JP, Millier C (1981) Amance 1981, manuel de la programmation statistique. CNRF, INRA

Barrière $Y$, Gallais A, Huguet L, Traineau R (1985) Le maïs brown-midrib plante entière. I. Caractéristiques générales. Bull Tech CRZV, Theix 60, 43-58

Barrière $Y$, Gallais A, Derieux M, Panouillé A (1987) Etude de la valeur agronomique au stade de récolte ensilage de différentes variétés de maïs grain sélectionnées entre 1950 et 1980 . Agronomie 7 , 73-79

Barrière Y, Emile JC, Traineau R (1988) Energy value of silage maize and zootechnical performances. Preliminary report of the study of genetic variability. Colloque CRA Gembloux, Belgique, novembre 1988, $14 \mathrm{p}$

Biston R, Dardenne P (1988) Applying NIRS to measure the silage maize in vitro and in vivo digestibility.
In : Proceedings quality of silage maize, digestibility and zootechnical performance, Gembloux (Belgique), 56-57

Byers FM (1974) The importance of associative effects of feeds on corn silage and corn grain net energy values. Ph D Thesis Colorado state Univ, Fort Collins

Chilliard Y, Rémond B, Agabriel J, Robelin J, Vérité R (1987) Variations du contenu digestif et des réserves corporelles au cours du cycle gestation lactation. Bull Tech CRZV Theix 70, 117-131

Daynard TB, Hunter RB (1975) Relationships among whole-plant moisture, grain moisture, dry matter yield and quality of whole-plant corn silage. Can $J$ PI Sci 49, 361-370

Deinum B (1987) Genetic and environmental variation in quality of forage maize in Europe. Miscellaneous paper of Agricultural University, Wageningen, The Netherlands, $18 p$

Deinum B, Bakker JJ (1981) Genetic differences in digestibility of forage maize hybrids. Neth J Agric Sci 29, 93-98

Deinum B, Struik PC (1985) Improving the nutritive value of forage maize. Maize and sorghum section of Eucarpia. In : Proceedings of the 13th congress, Wageningen, 9-12 September 1985, 77-90

Deinum B, Struik PC (1989) Genetic variation in digestibility of forage maize (Zea mays L) and its estimation by near infrared reflectance spectroscopy (NIRS). An analysis. Euphytica 42, 89-98

Demarquilly C (1969) Valeur alimentaire du maïs fourrage. 1. Composition chimique et digestibilité du maïs sur pied. Ann Zootech 18, 17-32

Derieux $M$, Darrigrand $M$, Gallais A, Barrière $Y$, Bloc D, Montalant $Y$ (1987) Estimation du progrès génétique réalisé chez le maïs grain en France entre 1950 et 1985. Agronomie 7, 1-11

Dulphy JP, Demarquilly C, Henry M (1975) Perte de composés volatils lors de la détermination à l'étuve de la teneur en matière sèche des ensilages. Ann Zootech 24, 743-756

Dulphy JP, Demarquilly C (1981) Problèmes particuliers aux ensilages. In: Prévision de la valeur nutritive des aliments des ruminants, INRA, 81-104

El-Shazy K, Dehority BA, Johnson RR (1961) Effect of starch on the digestion of cellulose in vitro and in vivo by rumen micro-organisms. J Anim Sci 20 , 268-273

Faverdin P, Hoden A, Coulon JB (1987) Recommandations alimentaires pour les vaches laitières. Bull Tech CRZV Theix, INRA 70, 133-152

Gallais A, Pollacsek M, Huguet I (1976) Possibilités de sélection du maïs en tant que plante fourragère. Ann Amélior Plant 26, 591-605

Gallais A, Vincourt P, Huguet $L$ (1981) Objectifs et critères de sélection du maïs fourrage. Congrès Eucarpia, Montreux, Suisse, septembre 1981

Grand C, Parmentier P, Boudet A, Boudet AM (1985) Comparison of lignins and of enzymes involved in lignification in normal and brown-midrib (bm3) mutant corn seedlings. Physiol Veg 23, 905-911

Grenet E, Barry P (1988) Dégradation microbienne dans le rumen du maiis normal et du maïs brownmidrib observée au microscope électronique à balayage. Reprod Nutr Dev 28, 125-126 
Hemken RW, Clark NA, Goering HK, Vandersall JH (1971) Nutritive value of corn silage as influenced by grain content. $J$ Dairy Sci $54,383-389$

Henning PA, van der Linden $Y$, Mattheyse ME, Nauhaus WK, Schwartz HM (1980) Factors affecting the intake and digestion of roughage by sheep fed maize straw supplemented with maize grain. $J$ Agric Sci Camb 94, 565-573

Hoden A, Barrière $Y$, Gallais A, Huguet L, Journet $M$, Mourguet A (1985) Le maïs brown-midrib plante entière. III. Utilisation sous forme d'ensilage par les vaches laitières. Bull Tech CRZV Theix 60, 43-58

Hoden A, Coulon JB, Faverdin P (1988) Alimentation des vaches laitières. In: Alimentation des bovins, ovins et caprins. INRA (sous la direction de R Jarrige), 135-158

ITEB (1988-89) Bulletin Ognoas Flash N. 12, 14, 16, 17

Jarrige R (1988) Alimentation des bovins, ovins et caprins. INRA, $471 p$

Joanning SW, Johnson DE, Barry BP (1981) Nutrient digestibility depressions in corn silage-corn grain mixtures feed to steers. J Anim Sci 53 (4), 10951103
Jung HG (1989) Forage lignins and their effects on fiber digestibility. Agron J 81, 33-38

Lila M, Barrière Y, Traineau R (1986) Mise au point et étude d'un test enzymatique de la digestibilité de fourrages pauvres ou riches en amidon. Agronomie 6, 285-291

Little TM, Hills FJ (1978) Subplot as repeated observations. In : Agricultural experimentation, design and analysis. John Wiley \& sons, 125-137

Malterre C, Bertin G, Gallais A, Huguet L, Micol D (1985) Le maïs brown-midrib plante entière. Il. Utilisation sous forme d'ensilage par les taurillons. Bull Tech CRZV Theix 60, 43-58

Rémond B, Journet M (1977) Utilisation de 2 ensilages de maïs de composition morphologique différente par les vaches laitières. Bull Tech CRZV Theix 29, 49-52

Struik PC (1983) Physiology of forage maize (Zea mays $L$ ) in relation to its production and quality. Thèse, Agricultural University of Wageningen, $252 \mathrm{p}$

Tilley JMA, Terry RA (1963) A two stage technique for the in vitro digestion of forage crops. $J$ Br Grassl Soc 18, 104-111 